\title{
Phase-induced atomic permutations in icosahedral quasicrystals: a model for self-diffusion
}

\author{
A Rüdinger and $\mathrm{H}-\mathrm{R}$ Trebin \\ Institut für Theoretische und Angewandte Physik, Universität Stuttgart, Pfaffenwaldring 57, \\ 70550 Stuttgart, Germany
}

Received 4 July 1994

\begin{abstract}
The process of phase-induced self-diffusion has been investigated for the threedimensional Ammann-Kramer-Penrose tiling. This happens along the lines of Kalugin and Katz for the octagonal planar tiling. It is found that for any permutation within two subsets of the ten vertices in a triacontahedral cage there is a corresponding loop in phase space. There are other loops by which vertices are exchanged between interlocked triacontahedral cages. Along chains of such triacontahedral cages, percolative diffusion is possible. This self-diffusion, however, occurs along two separated sublattices. Thus it is a two-component diffusion.
\end{abstract}

\section{Introduction}

Quasicrystals carry the additional degree of freedom of the 'phase', as do incommensurably modulated crystals. In the latter the phase describes the relative position of the periodic basic structure and the incommensurate modulation wave. A change of phase in a quasiperiodic tiling causes a rearrangement of the tiles due to flips of selected vertices. If a tiling is constructed by the projection method or by the method of atomic surfaces, the phase denotes the position of the strip or the intersection point of parallel space $E$ in orthogonal space $E^{\prime}$, respectively. In a seminal paper Frenkel et al [1] have shown for the octagonal AmmannBeenker tiling [2] that, if certain closed loops are traversed by the phase in $\boldsymbol{E}^{\prime}$, the tiling returns to its original form, but with permutated atomic positions. These permutations have been viewed by Katz and Kalugin [3] as elementary steps in an atomic transport process, which at high temperatures drastically enhance the self-diffusivity. Hints for this phenomenon are coming from deformation experiments on quasicrystals: at about $80 \%$ of the absolute melting temperature the mechanical behaviour of quasicrystals changes from brittleness to ductility [4-6]. Evidently, at high temperatures plastic deformation occurs in quasicrystals through dislocation motion [7]. However, as dislocations are partials in quasicrystals, they drag along a stacking fault, i.e. a wall where the local phase is jumping. Hence the dislocations are greatly impeded in their mobility, unless the stacking fault is able to dissolve through self-diffusion.

In this paper we study a possible mechanism for the self-diffusion process in the icosahedral Ammann-Kramer-Penrose tiling, which is a model for icosahedral quasicrystals [8]. We first repeat the geometry of the elementary atomic transport in the octagonal tiling and generalize it to the three-dimensional case. For good visualization the projection method and the method of atomic surfaces are employed. 


\section{Self-diffusion in the octagonal tiling}

In the standard planar octagonal Ammann-Beenker tiling [2], the acceptance domain is a regular octagon. The atomic surfaces consist of the same octagons, attached to the lattice points of a four-dimensional cubic lattice. They are oriented parallel to $E$, and are connected by 'steps' parallel to $E^{\prime}$.

A frequent subpattern in $E$ is an octagonal cage, containing three vertices, such that the octagon is divided into two squares and four $45^{\circ}$-rhombi (figure 1). The three vertices belong to a small octagon inside the cage. In the canonical mapping $\varphi$ from $E$ to $E^{\prime}$
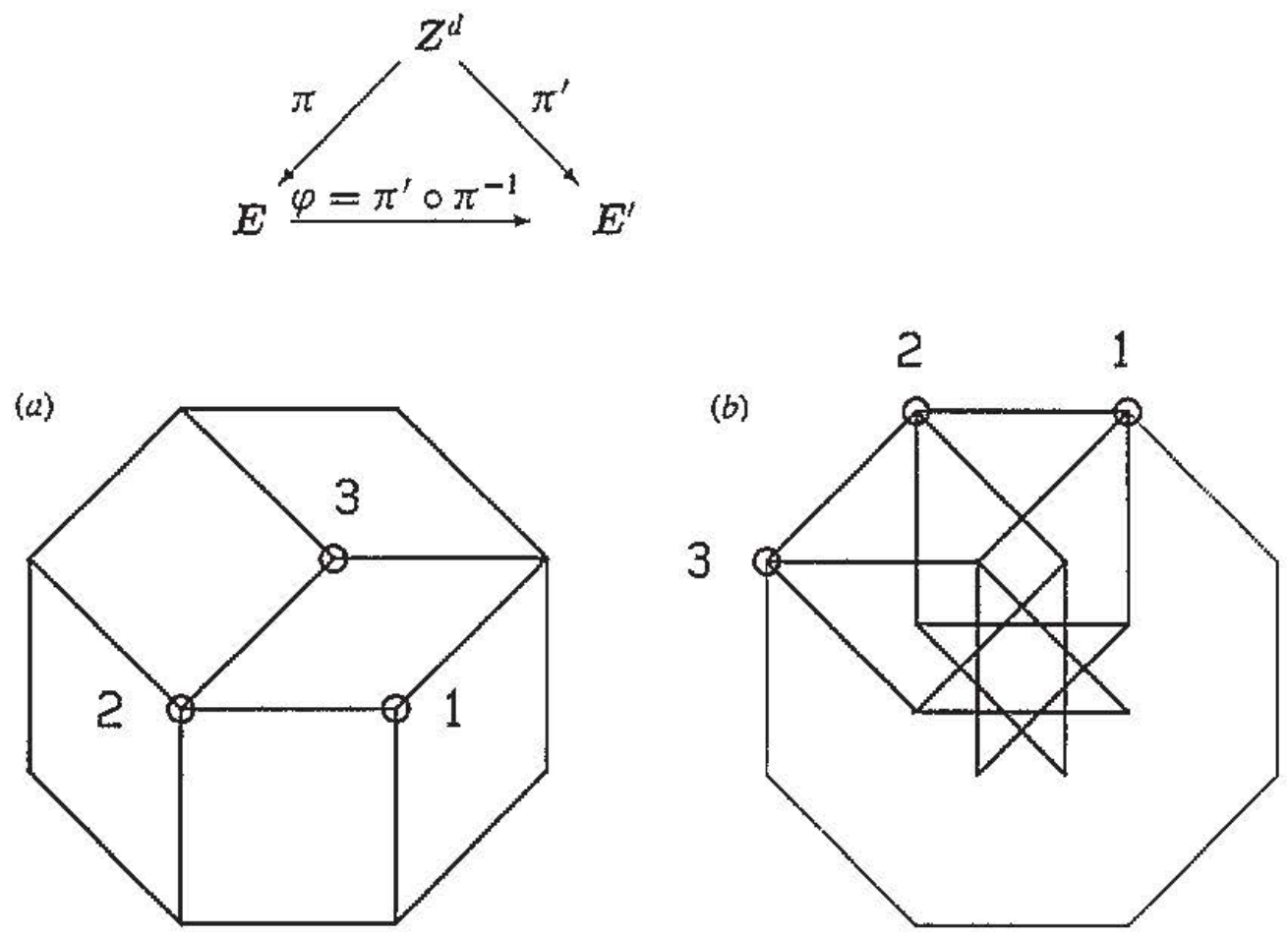

Figure 1. Decomposition of the octagon in $E(a)$ and its dual in $E^{\prime}(b)$.

with $d=4$, the two concentric octagons in $E$ are also mapped into two concentric octagons in $E^{\prime}$, but with the internal points becoming external ones and vice versa. In figure 1 the set $M$ of the vertices of the decomposed octagon in $E$ and the dual set $M^{\prime}=\varphi(M)$ in $E^{\prime}$ are depicted (concerning the basis vectors, $\mathrm{cf}$ [9]). For the sake of clarity the bonds of the vertices in $E$ are also drawn in $E^{\prime}$. The acceptance domain in $E^{\prime}$ is marked by thin lines. The images of three internal vertices in $E$ are lying arbitrarily closef to the boundary of the acceptance domain in $E^{\prime}$. They are occupying adjacent positions, so that $M^{\prime}$ can be moved into two linearly independent directions without leaving the acceptance domain. This is a necessary and sufficient condition for the occurrence of the corresponding subpattern $M$ in a perfect tiling. By an infinitesimal change of the phase one of these marginal points can leave the acceptance domain, and another one, separated by three edges, enters. Hence in $E$ a flip does occur for one of the internal vertices, whereas the vertices of the exterior cage remain untouched (figure 2). As can be seen in $E$ and in $E^{\prime}$, two of the three internal vertices ( 1 and 3 ) are able to flip.

In the representation of atomic surfaces, the octagonal surfaces belonging to the eight internal positions of $E$ are arranged as in figure 3, sharing a common vertex. The loop

$f$ In the figures, the points that are marked on the boundary are supposed to lie inside the acceptance domain within an $\epsilon$-neighbourhood of its boundary. 

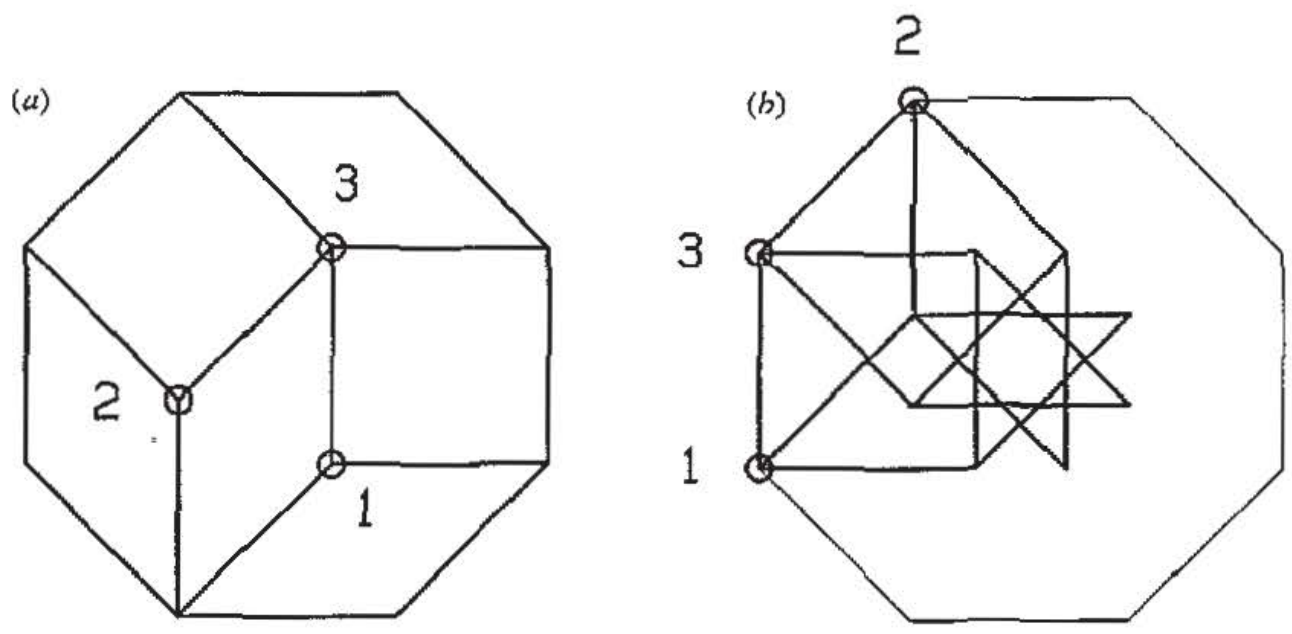

Figure 2. The octagon in $E(a)$ and its dual in $E^{\prime}(b)$ after one flip.

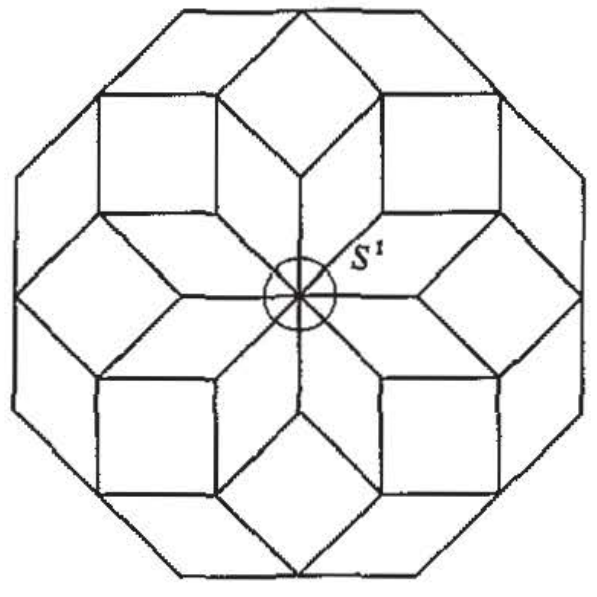

Figure 3. Eight atomic surfaces, belongitg to the eight internal vertices of the octagonal cage.

encircling this vertex, denoted by $S^{1}$, is responsible for the elementary transport process.

A single atomic octagon in $E^{\prime}$ corresponds to one of the vertices inside the octagonal cage. It cuts a sector of $135^{\circ}$ out of the loop $S^{l}$. We denote this sector 'influence domain of the vertex', because the vertex is occupied in $E$ as long as the phase is contained in this domain. The atomic octagons altogether cut out eight sectors of $45^{\circ}$. Each sector is being shared by three influence domains, belonging to the three internal vertices in $E$. If the phase crosses the border of a sector, it changes from one domain to another, whereupon a flip happens in the octagonal cage. After the loop has been traversed, eight flips have occurred, taking the subpattern back to its original form, but permuting three atoms.

This behaviour is due to the topological structure of the phase space, which can be described in terms of covering spaces. (For a treatment of covering spaces, of $[10,11]$.) Each of the eight atomic octagons shares two edges with two other octagons. If these edges are identified, a three-sheeted local covering of $E^{\prime}$ results, with the central vertex becoming a branch point. The covering is sketched in figure 4 , where the connection of the upper and lower atomic octagon is omitted for the sake of clarity. It consists of eight patches which, projected to the base space, locally yield a decomposition of the latter into eight sectors. The eight patches correspond to the eight internal positions of an octagon in $\boldsymbol{E}$, and the eight sectors to the eight possible decompositions of an octagon. The fibre $p^{-i}\left(x_{\text {phase }}\right)$ consists of three points, corresponding to the three internal vertices of an octagon in the tiling. 

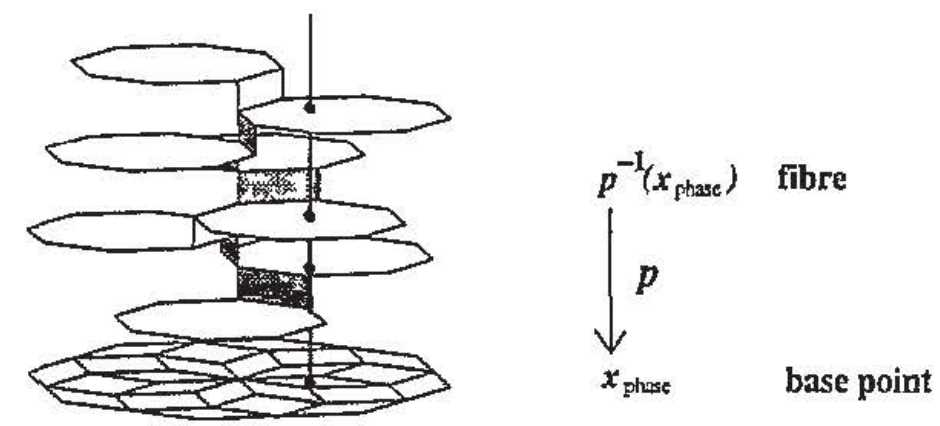

Figure 4. Three-sheeted covering of the neighbourhood of a vertex in $E^{\prime}$ and its projection to the base space.

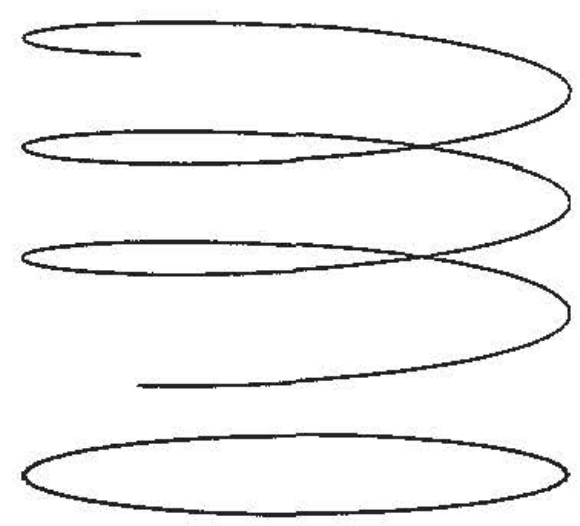

Figure 5. Three-sheeted covering of $S^{l}$.

The restriction of the covering to $S^{l}$ contains all the local information of the covering near a branch point and is depicted schematically in figure 5, where again the open ends have to be identified.

If $x_{\text {phase }}$ travels along a loop encircling the branch point, the result is a permutation of the internal vertices of an octagon, as described in [1]. We characterize the loops by elements of the fundamental group $\pi_{1}\left(S^{1}, x_{0}\right)=Z$ of the circle. The integer assigned to each loop is the winding number. We can construct a homomorphism $f$ from $\pi_{1}\left(S^{1}, x_{0}\right)$ to the permutation group of three objects, $S_{3}$. This homomorphism is given explicitly by

$$
\begin{aligned}
& f: \pi_{1}\left(S^{1}, x_{0}\right) \mapsto S_{3} \\
& \mathbf{Z} \ni n \mapsto f(n)=\sigma^{n} \in S_{3} \\
& \text { with } \sigma=(123) \in S_{3} .
\end{aligned}
$$

The fact that a loop of winding number 1 does lead to a 3-cycle in $S_{3}$ can also be seen by considering the eight flips in $E^{\prime}$ (cf figures $1(b)$ and $2(b)$, where the first flip is shown). The representation of the flips according to figures $1(b)$ and $2(b)$ will be especially helpful for the icosahedral case.

In figure 6 two interlocked octagonal cages in $\boldsymbol{E}$ are displayed, their internal octagons being attached and sharing two edges.

The 14 possible positions in $E$ correspond to 14 atomic octagons in $E^{\prime}$, which are grouped around two vertices, separated by a basis vector. The local neighbourhood of these two vertices can be tested by two connected circles $S^{1} \# S^{1} \simeq S^{1}$, sketched in figure 7 .

In analogy with the above example, a five-sheeted covering of $S^{1}$ results, consisting of 14 patches, the projection of which yields a decomposition of the base space into 14 sectors. Therefore there are 14 possibilities of occupying five of the 14 internal positions. Encircling both vertices is equivalent to traversing a loop around one vertex first, then 


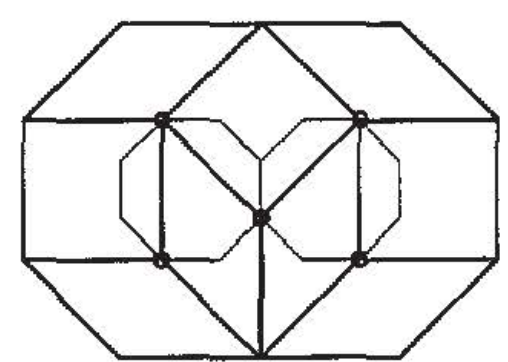

Figure 6. 'Two interlocked octagons with internal octagons being attached.

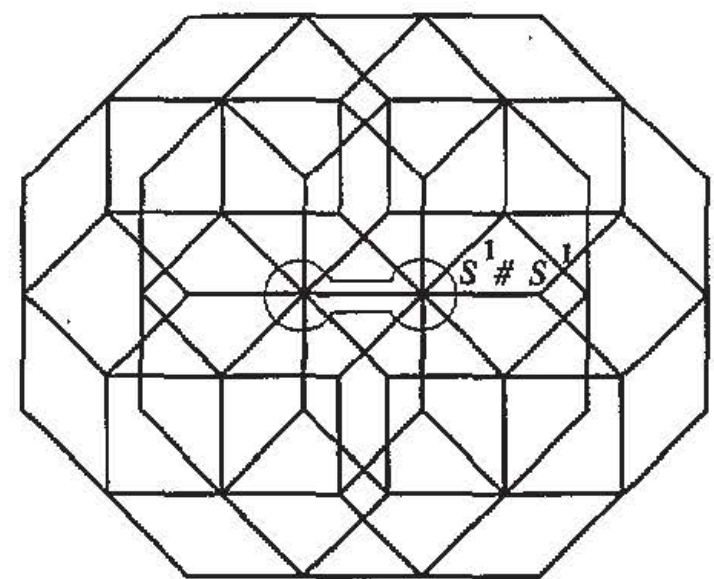

Figure 7. 14 atomic surfaces arranged around two vertices.

moving to the second, encircling it and returning. In this process a separate permutation of three atoms occurs in each internal octagon and, as a result, a cyclic permutation of the five vertices takes place in the double cage. In [12] it is shown how the ring can be extended to infinite size up to a percolation limit. We are now going to extend these considerations to a three-dimensional quasicrystal.

\section{Remarks on the icosahedral Ammann-Kramer-Penrose pattern}

In the icosahedral quasicrystal as defined in [13], the acceptance domain is a triacontahedron, a polyhedron with 12 five-fold and 20 three-fold vertices, which displays icosahedral symmetry. This triacontahedron is the convex hull of the six icosahedral vectors $e_{t}^{f}$ in $E^{\prime}$. In physical space $E$, the corresponding vectors $e_{i}$ analogously form a triacontahedral cage, which occurs frequently in the icosahedral tiling. In the inner part of this cage 32 possible vertex positions are located, ten of which are occupied in a perfect tiling. The triacontahedron is divided into ten prolate and ten oblate Ammann rhombohedra, the elementary tiles of the icosahedral quasicrystal. It is worth mentioning that this decomposition has already been realized in the thirties [14]. In order to visualize the decomposition, we consider the canonical mapping $\varphi$ from $E$ to $E^{\prime}$, as shown above with $d=6$. It maps the 32 vertices of the triacontahedron in $E$ into the 32 internal vertices of the triacontahedron in $E^{\prime}$ and vice versa. A subpattern $M$ occurs in the perfect tiling if its dual $M^{\prime}=\varphi(M)$ can be moved within the acceptance domain into three linearly independent directions.

In figure 8 we are looking at the acceptance domain along a two-, three- and fivefold axis, respectively. The ten internal vertices in $E$ are mapped onto ten vertices of the triacontahedron in $E^{\prime}$; they are marked by a circle. The dual pattern can be moved within the acceptance domain, and therefore the corresponding decomposition of the triacontahedron in $E$ occurs with a finite frequency. The ten vertices form two disjoint sets: three of them belong to the 12 five-fold vertices and seven to the 20 three-fold ones of the triacontahedron in $E^{\prime}$.

The decomposition does not display any symmetry, so that, in a perfect tiling, there are 120 possibilities of occupying 10 of the 32 vertices inside a triacontahedral cage. They form two orbits under the proper icosahedral point-group $\mathcal{Y}$, which are connected by a single flip. 

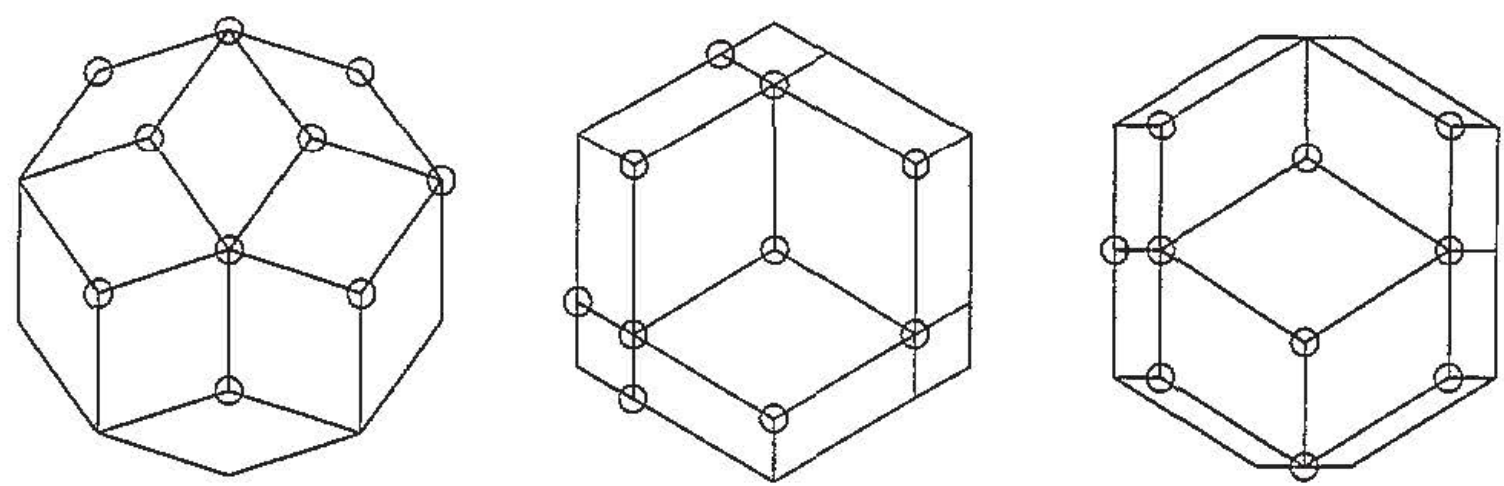

Figure 8. Occupied vertices of the triacontahedron in $E^{\prime}$.

There are 40 additional possibilities of arranging the 20 Ammann rhombohedra inside the triacontahedral cage, but these do not occur in a perfect tiling (cf Lück in [15]).

\section{Self-diffusion in the icosahedral Ammann-Kramer-Penrose pattern}

\subsection{A single triacontahedral cage}

In the representation of atomic surfaces, 32 atomic triacontahedra belonging to the internal positions of the triacontahedral cage in $E$ are attached to a central point (compare with figure 3 of the octagonal case). As, for the moment, our interest is directed towards the local properties of the extended atomic surface, we surround the central point by a small sphere $S^{2}$. 12 triacontahedra, which are attached to the central point by a five-fold vertex, cut spherical pentagons out of this sphere; 20 remaining ones, being attached by a three-fold vertex, are spherical triangles. Each of these 32 patches is an influence domain of one of the internal positions. Altogether they form a ten-sheeted covering of $S^{2}$, since 10 of the 32 internal vertices of the triacontahedron are occupied for any generic phase $x_{\text {phase }} \in S^{2}$. Projected to the base space, the 32 patches do overlap and yield a decomposition of $S^{2}$ into 120 spherical triangles. The resulting graph on $S^{2}$ is equivalent to that of a polytope with 62 vertices, 180 edges and 120 triangular faces, the dual of a truncated icosidodecahedron. The 62 vertices form three orbits under $\mathcal{Y} .12$ of them have five-fold symmetry, 20 have three-fold and the remaining 30 have two-fold symmetry. Each of the 120 faces on the sphere $S^{2}$ represents one arrangement of the ten internal vertices of the triacontahedron in $E$. If we move $x_{\text {phase }}$ across a boundary (one of the 180 edges), another decomposition of the triacontahedron is obtained, which differs from the initial one by a single flip. (For an illustration of a single flip in the tiling space, see [16].) Since the faces are triangles, three of the ten internal vertices of the triacontahedron are able to flip, starting from a given configuration.

In figure $9(a)$ an elementary flip is depicted in $E^{\prime}$. The decagon is the silhouette of the acceptance domain along a five-fold axis. On an infinitesimal change of the phase in a suitable direction, vertex 1 is moving out of the acceptance domain. The new vertex is like the old one, a five-fold vertex. Examination of the other three possible flips shows that three-fold vertices always remain three-fold ones when being flipped, and so do the five-fold ones. As for each generic $x_{\text {phase }} \in S^{2}$, three five-fold vertices and seven three-fold vertices are occupied, and since flipping does not change the character of a vertex (five- or three-fold), the ten-sheeted covering of $S^{2}$ contains two disconnected parts: a seven-sheeted covering, consisting of 20 triangular patches, and a three-sheeted one, consisting of 12 pentagonal patches. 


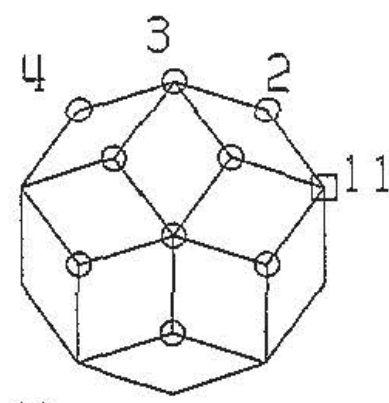

(a)

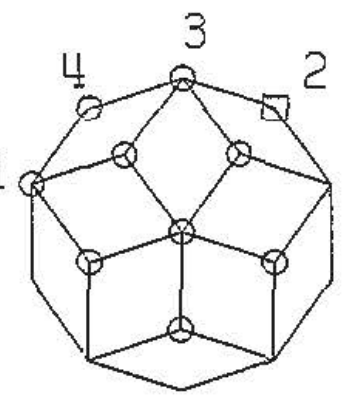

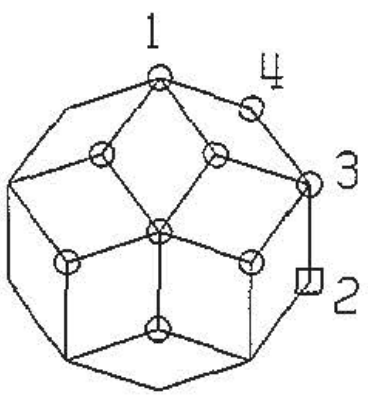

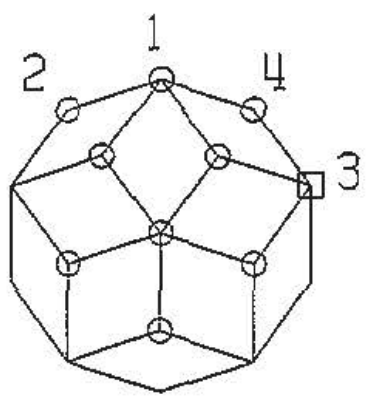

(b)

Figure 9. (a) First and (b) last flip occurring on a loop of $x$ phase around a branch point.

One would think that all of the 62 vertices on $S^{2}$ are branch points. 50 of them, however, are merely intersections of edges of patches in the projection to the base space. Only the points, where more than two patches touch each other, i.e. the vertices of the patches, are branch points of the two coverings. Hence the branch points are just the 12 icosahedral vertices on $S^{2}$, the set of which will be denoted $B$. Moving the phase through one of these branch points will be avoided for the sake of unambiguity.

If $x_{\text {phase }}$ is traversing a loop $\gamma$ in $S^{2} \backslash B$, a step-by-step substitution of one vertex by another takes place. A given vertex does not disappear unless $x_{\text {phase }}$ leaves the influence domain of the vertex, i.e. a spherical pentagon for a five-fold vertex, and a spherical triangle for a three-fold vertex. When $x_{\text {phase }}$ returns to its initial position, the tiling obtains its original form, but with permutated atomic positions.

Loops $\gamma$ in $S^{2} \backslash B$ with base point $x_{0}$ can be lifted to the two coverings and lead to automorphisms of the two fibres $p_{3}^{-1}\left(x_{0}\right)$ and $p_{7}^{-1}\left(x_{0}\right)$ :

$$
\begin{aligned}
& f: \pi_{1}\left(S^{2} \backslash B, x_{0}\right) \mapsto S_{3} \times S_{7} \\
& \gamma \mapsto\left(f_{3}(\gamma), f_{7}(\gamma)\right) .
\end{aligned}
$$

Figure 9 shows the first and the last flip induced by a loop of $x_{\text {phase }}$ around a branch point; vertices marked by a square are the next ones to flip.

The initial and final positions of the occupied vertices are identical; atoms 1 and 3 , however, have exchanged their places and so have atoms 2 and 4 . Hence a loop $\gamma$ encircling a branch point maps to the direct product of two transpositions in $S_{3} \times S_{7}$ :

$$
\begin{aligned}
& f_{3}(\gamma) \in\left(2^{1} 1^{1}\right) \subset S_{3} \\
& f_{7}(\gamma) \in\left(2^{1} 1^{5}\right) \subset S_{7} .
\end{aligned}
$$

$\left(\left(k_{1}^{n_{1}}, k_{2}^{n_{2}}, \ldots, k_{i}^{n_{i}}\right)\right.$ denote the cycle decomposition of an element in the permutation group $S_{n_{1}} \cdot k_{i}+\ldots+n_{i} \cdot k_{i}$. A conjugate class is formed by elements of the same cycle structure.)

Encircling two branch points corresponds to the class-product in $S_{3} \times S_{7}$. It depends on the relative position of the two branch points, which actually describes the permutation of the resulting class. This holds equally well for loops surrounding more than two branch points: some loops and their corresponding cycles in $S_{3}$ and $S_{7}$ are shown in figure 10 .

We now want to prove that all elements in $S_{3} \times S_{7}$ can be obtained by loops $\gamma \in$ $\pi_{1}\left(S^{2} \backslash B, x_{0}\right)$. This amounts to showing that the homomorphism $f: \pi_{1}\left(S^{2} \backslash B, x_{0}\right) \mapsto S_{3} \times S_{7}$ is surjective. The map $f$ can be divided into two homomorphisms $f_{3}$ and $f_{7}$ according to (5). In a first step we will show that these homomorphisms are surjective, and in a second step, that this holds equally for the composed homomorphism $f$. 


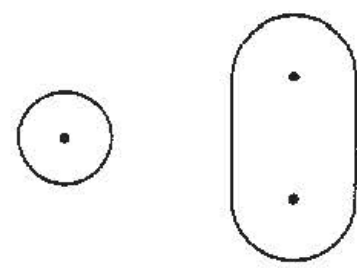

$2^{1} 1^{1}$

$2^{1} 1^{5}$

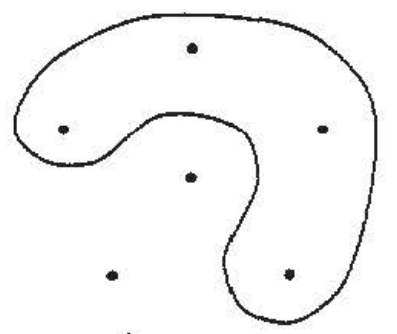

$3^{1}$

$51^{1}$

$3^{1}$

$2^{2} 1^{3}$

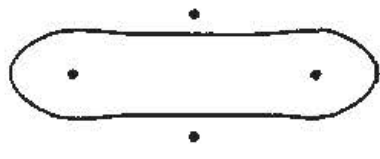

$1^{3}$

$3^{x} 1^{4}$

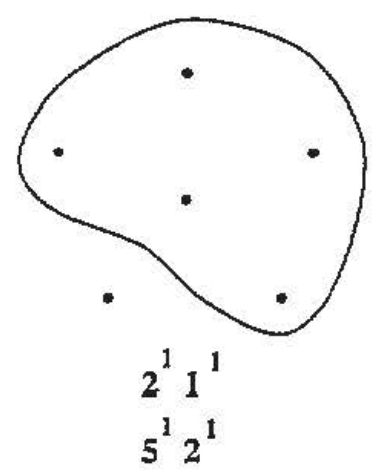

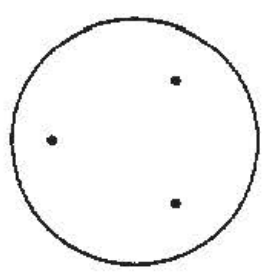

$2^{1} 1^{1}$

$2^{1} 1^{5}$

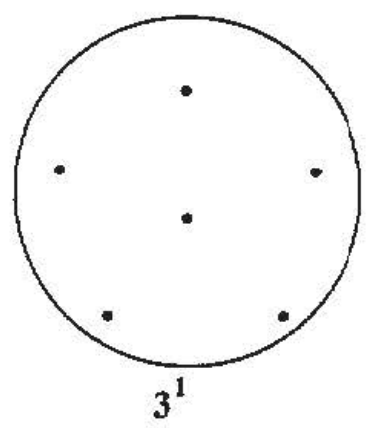

$3^{1} 2^{2}$

Figure 10. Loops in $S^{2} \backslash B$ and the corresponding cycles in $S_{3} \times S_{7}$.

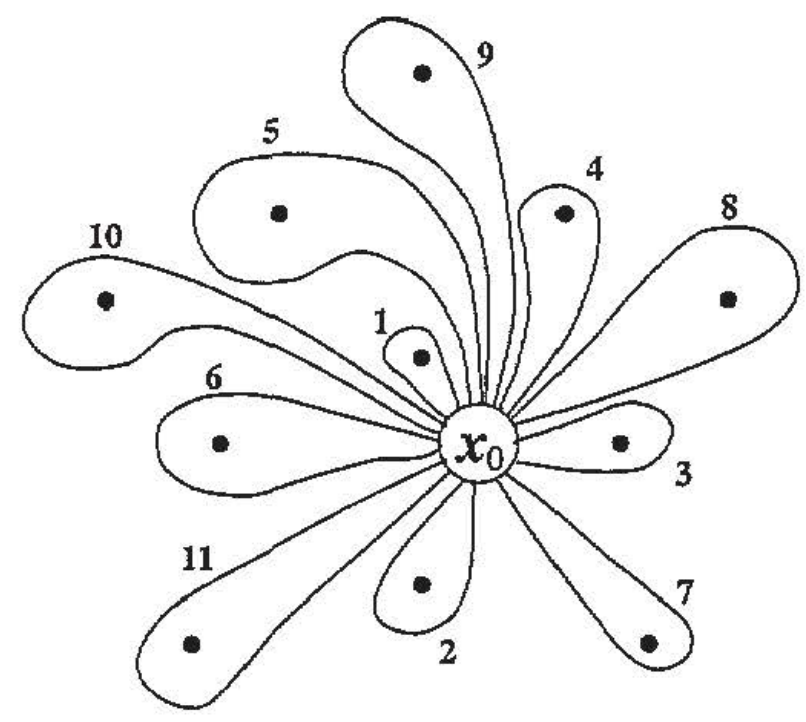

Figure 11. The 11 generators $g_{i}$ of $\pi_{1}\left(S^{2} \backslash B, x_{1}\right)$.

The fundamental group $\pi_{1}\left(S^{2} \backslash B, x_{0}\right)$ is the free group of 11 generators $g_{i}(i=1 \ldots 11)$, which are chosen as indicated in figure 11 (the loop encircling the 12th branch point is the sum of the 11 generators).

Each generator maps to a transposition both in $S_{3}$ and in $S_{7}$, which is illustrated in figure 12.

Since a generative base of the symmetric group $S_{n}$ is formed by $n-1$ consecutive transpositions (12), (23), .., $(n-1, n)$, the sets $f_{3}\left(g_{i}\right)$ and $f_{7}\left(g_{i}\right)$ are generative in $S_{3}$ and $S_{7}$, respectively. Therefore the homomorphisms $f_{3}$ and $f_{7}$ are surjective.

In order to show that the composed homomorphism $f$ is surjective as well, we first remark that two loops in the kernel of $f_{7}$ can be found, so that their image under $f_{3}$ 

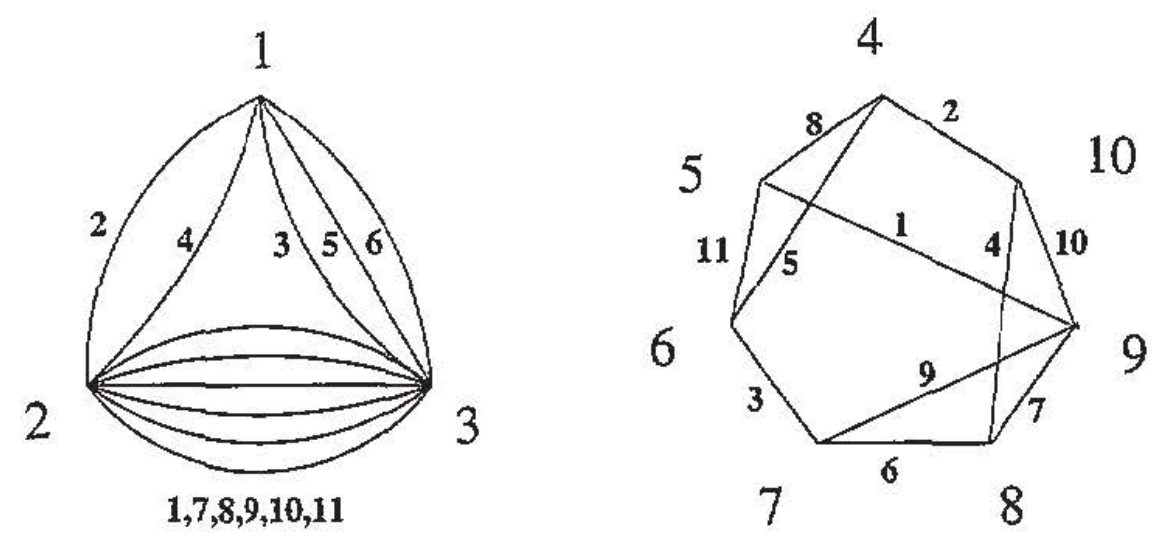

Figure 12. Transpositions in $S_{3}$ and $S_{7}$, induced by the generators $g_{i}$.

provides a generative base in $S_{3}$ :

$$
\begin{array}{ll}
g_{5} g_{11} g_{8} \in \operatorname{Ker} f_{7} & f_{3}\left(g_{5} g_{11} g_{8}\right)=(13) \\
g_{4} g_{10} g_{7} \in \operatorname{Ker} f_{7} & f_{3}\left(g_{4} g_{10} g_{7}\right)=(12) .
\end{array}
$$

This result shows that the homomorphism $f$ is surjective, as can be seen as follows.

Take an arbitrary permutation $\left(\sigma_{3}, \sigma_{7}\right) \in S_{3} \times S_{7}$. Since $f_{7}$ is surjective, $\gamma_{7} \in f_{7}^{-1}\left(\sigma_{7}\right)$ can be chosen, which yields $f\left(\gamma_{7}\right)=\left(f_{3}\left(\gamma_{7}\right), f_{7}\left(\gamma_{7}\right)\right)=\left(f_{3}\left(\gamma_{7}\right), \sigma_{7}\right)$. Because Ker $f_{7}$ is generative in $S_{3}$, a loop $\gamma_{3}$ with $f_{3}\left(\gamma_{3}\right)=\left[f_{3}\left(\gamma_{7}\right)\right]^{-1} \sigma_{3}$ can be composed by elements of $\operatorname{Ker} f_{7}$. Then $\gamma_{7} \gamma_{3}$ is a loop which yields the desired permutation $\left(\sigma_{3}, \sigma_{7}\right)$ :

$$
\begin{aligned}
f\left(\gamma_{7} \gamma_{3}\right) & =\left(f_{3}\left(\gamma_{7} \gamma_{3}\right), f_{3}\left(\gamma_{7} \gamma_{3}\right)\right) \\
& =\left(f_{3}\left(\gamma_{7}\right) f_{7}\left(\gamma_{3}\right), f_{7}\left(\gamma_{7}\right) f_{7}\left(\gamma_{3}\right)\right) \\
& =\left(f_{3}\left(\gamma_{7}\right)\left[f_{3}\left(\gamma_{7}\right)\right]^{-1} \sigma_{3}, \sigma_{7} \mathrm{Id}\right) \\
f\left(\gamma_{7} \gamma_{3}\right) & =\left(\sigma_{3}, \sigma_{7}\right) .
\end{aligned}
$$

Thus all permutations in $S_{3} \times S_{7}$ can be obtained by loops in $S^{2} \backslash B$.

\subsection{Two interlocked triacontahedral cages}

Loops of the phase $x_{\text {phase }}$ in the neighbourhood of a vertex in $E^{\prime}$ have been shown to yield all possible permutations within the two sets of internal vertices of a triacontahedral cage. In the following, larger loops than the ones treated above will be considered. These lead to an exchange of atoms between interlocked triacontahedra.

The 32 internal positions of a triacontahedral cage form two orbits under $\mathcal{Y}$, namely the vertices of an icosahedron and those of a dodecahedron, respectively. Both polyhedra are concentric and their edges have the same lengths.

In the case of two interlocked triacontahedra, the dodecahedron inside one triacontahedral cage has five vertices in common with the icosahedron inside the other cage; more precisely, a face of the dodecahedron coincides with the 'base-face of the cap' of the icosahedron, as depicted in figure 13. (This is the icosahedral analogue to the two small octagons in figure 6. )

Therefore there are $2 \times(20+12-5)=54$ internal positions within two interlocked triacontahedral cages. In the dual space $E^{\prime}$, the internal positions of each triacontahedral cage are mapped onto a triacontahedron (figure 8). Since ten positions belong to the interior of both of the two triacontahedral cages in $E$, the triacontahedra in $E^{\prime}$ do intersect along 


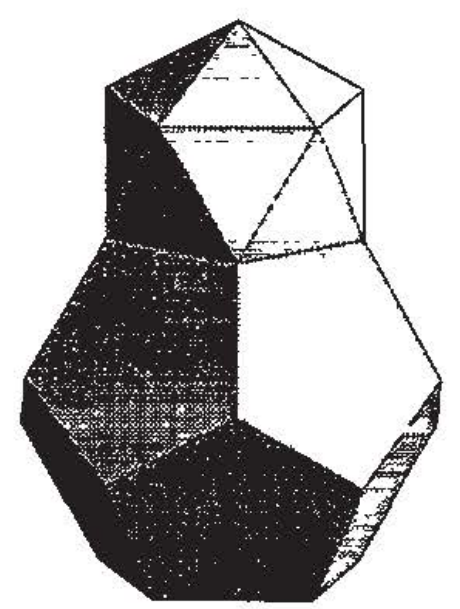

Figure 13. Dodecahedron and attached icosahedron.

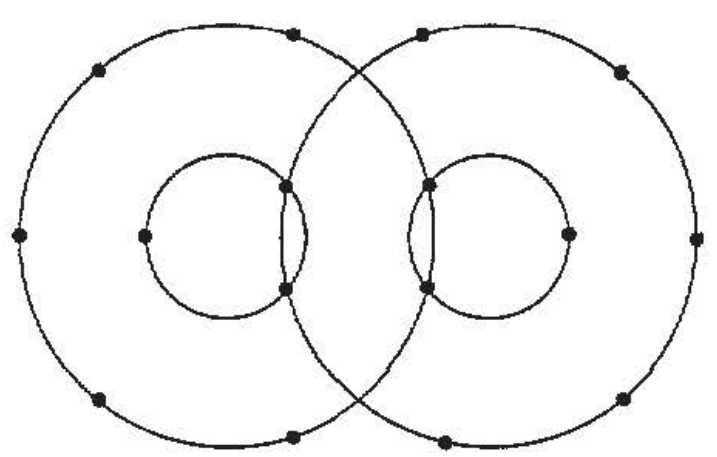

Figure 14. Internal points inside two interlocked triacontahedra.

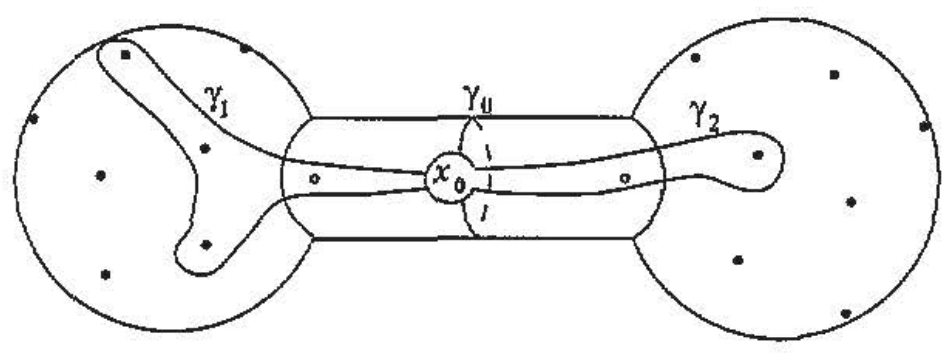

Figure 15. $S^{2} \# S^{2}$ with loops on it.

a decagonal silhouette. Four of these 10 common positions are occupied by vertices (cf figure 8). Therefore there remain $2 \times 10-4=16$ internal vertices within the two interlocked triacontahedral cages. The positions of these 16 vertices are sketched schematically in figure 14 , the two small circles representing icosahedra, the large ones dodecahedra.

In the representation of atomic surfaces, it can be calculated how many possibilities of occupying the 54 internal positions with 16 vertices occur in a perfect tiling.

The 54 possible positions in $E$ correspond to 54 atomic triacontahedra, which are grouped around two vertices, separated by a basis vector. The local neighbourhood of these two vertices can be tested by two connected spheres $S^{2} \# S^{2} \simeq S^{2}$, which are sketched in figure 15.

Considerations analogous to those of testing one vertex by a sphere $S^{2}$ yield a graph on $S^{2} \# S^{2}$ equivalent to that of a polytope with 122 vertices, 350 edges and 230 faces. Therefore there are 230 possibilities of occupying 16 of the 54 internal positions in a perfect tiling. On each sphere $S^{2}$ there are 12 branch points, one of which is eliminated from each sphere by constructing the connected sum $S^{2} \# S^{2}$. Hence there remain $2 \times(12-1)=22$ branch points on $S^{2} \# S^{2}$, the set of which will be denoted $\tilde{B}$. Loops in $S^{2} \# S^{2} \backslash \tilde{B}$ induce permutations in $S_{8} \times S_{8}$. Starting the phase $x_{\text {phase }}$ on a point of the belt and traversing the 'belt-loop' $\gamma_{0}$ (figure 15) yields two transpositions within the four common points, which are depicted in figure 16.

If, in a first step, the phase $x_{\text {phase }}$ is moved along a loop encircling branch points on only one sphere (e.g. $\gamma_{1}$ in figure 15), the vertices within one triacontahedral cage are permutated; vertices which only belonged to one triacontahedral cage may become common vertices of both cages. In a second step these vertices can be transferred out of the domain common to both cages into the second triacontahedral cage by moving $x_{\text {phase }}$ along a loop around branch points on the second sphere (e.g. $\gamma_{2}$ ). Thus any vertex of one triacontahedral cage 


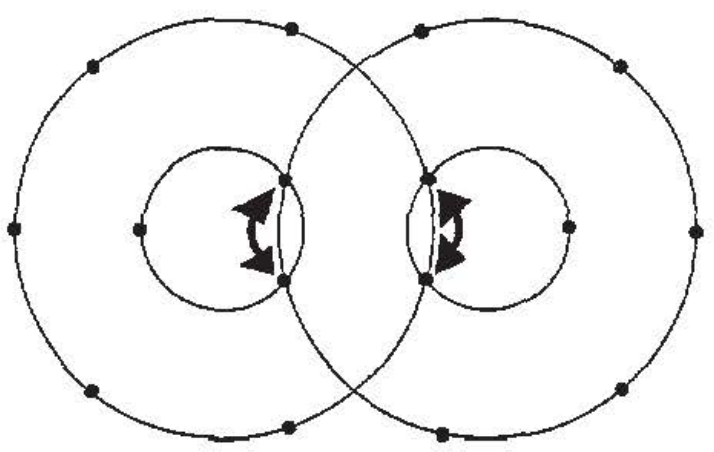

Figure 16. Transposition induced by transversing the 'belt-loop'.

can diffuse by elementary flips to the adjacent one.

\subsection{Global view and percolation limit}

Permutations of atomic positions within limited regions have been discussed by restricting the covering of the three-dimensional perpendicular space $E^{\prime}$ with triacontahedra to a covering of a two-dimensional testing surface. For a global view, the three-dimensional covering has to be considered. The branch lines of this covering are the edges of the atomic triacontahedra. The set of all branch lines forms a connected infinite graph of icosahedral basis vectors. The subset of icosahedral vectors encircled by a loop of the phase is generally not connected. Each connection component corresponds to one region in the tiling within which atomic permutations occur. In a small loop, permutations only occur within separated triacontahedral cages. Enlarging the size of the loop leads to an increase of the number density of these triacontahedral cages. At a certain threstald an exchange of atoms between interlocked triacontahedral cages becomes possible. Further increases of the loop size yield larger and larger clusters of interlocked triacontahedral cages, between which exchange of atoms is possible. If an infinite chain of icosahedral vectors is encircled by the loop, the percolation limit is obtained, and infinite clusters appear, thus permitting long-distance self-diffusion.

In contrast to the octagonal case, the self-diffusion in the icosahedral Ammann-KramerPenrose tiling is a two-component diffusion: the lattice $Z^{6}$ is the union of two icosahedrally invariant sublattices, $Z_{\text {even }}^{6}$ (which is the root lattice $D_{6}$ ) and $Z_{\text {odd }}^{6}$, with

$$
Z_{\text {even(odd) }}^{6}=\left\{x \in Z^{6} \mid \sum_{i=1}^{6} x_{i} \text { even(odd) }\right\} .
$$

Hence there are two disjoint sets of atomic positions in the tiling: $\pi\left(Z_{\text {even }}^{6}\right)$ and $\pi\left(Z_{\text {odd }}^{6}\right)$. Whereas the set $\pi\left(Z^{6}\right)$ displays a $\tau^{3}$ inflation behaviour, $\pi\left(Z_{\text {even }}^{6}\right)$ is invariant through the dilatation of ratio $\tau$ [17]. It can be shown that flips do not lead to transitions between $\pi\left(Z_{\text {even }}^{6}\right)$ and $\pi\left(Z_{\text {odd }}^{6}\right)$. This means that the covering of $E^{\prime}$ with atomic triacontahedra consists of two disconnected pieces and that phase-induced atomic permutations take place separately in two subquasilattices.

\section{Summary and outlook}

Up to now, global uniform phason shifts have been discussed. These are unlikely to be the physical mechanism for diffusion in quasicrystals. If we replace the global phase shift by a local one, all considerations concerning the permutation of atoms remain valid, but matching rule violations appear during the shift of the phase. By attributing an activation energy to 
these matching rule violations and to the barrier for the elementary flips, a temperaturedependent probability for the corresponding self-diffusion process can be established, similar to the case of an octagonal lattice [18], though the phase structure of a three-dimensional model might be more complicated.

\section{References}

[1] Frenkel D M, Henley C L and Siggia E D 1986 Topological constraints on quasicrystal transformations Phys. Rev. B $343649-69$

[2] Grünbaum B and Shephard G C 1987 Tilings and Patterns (New York: Freeman)

[3] Kalugin P A and Katz A 1993 A mechanism for self-diffusion in quasi-crystals Europhys. Lett. 21 121-6

[4] Tsai A P et al 1992 Temperature dependence of hardness and expansion in an icosahedral Al-Pd-Mn alloy Japan. J. Appl. Phys. 31 2530-1

[5] Shibuya T, Hashimoto T and Takeuchi S 1990 Plastic deformation of Al-Ru-Cu icosahedral quasicrystals Japan. J. Appl. Phys, 29 L349-51

[6] Bresson L and Gartias D 1993 Plastic deformation in AlCuFe icosahedral phases J. Non-Cryst. Solids 153, 154468

[7] Wollgarten $M$ et al 1993 Direct evidence for plastic deformation of quasicrystals by means of a dislocation mechanism Phys, Rev. Lett 71 549-52

[8] Kramer P 1985 On the theory of a non-periodic quasilartice associated with the icosahedral group Z Naturf. a $40775-88$

[9] Socolar J E S 1989 Simple octagonal and dodecagonal quasicrystals Phys. Rev. B 39 10519-51

[10] Jänich K 1990 Topologie (Berlin: Springer)

[11] Massey W S 1991 A Basic Course in Algebraic Topology (Berlin: Springer)

[12] Trub A and Trebin H-R 1994 Topology of the phase space, phase singularities, and diffusive motion in octagonal quasicrystals $J$. Physique I to be published

[13] 'Kutz A and Duneau M 1986 Quasiperiodic patterns and icosahedral symmetry J. Physique 47 181-96

[14] Kowalewski G 1938 Der Keplersche Kijrer und andere Bauspiele ed K F Koehlers (Leipzig: Antiquarium)

[15] Janot C and Dubois J M (eds) 1988 Proc. ILLCodest Workshop (Singapore: World Scientific)

[16] Katz A 1987 Some local properties of the three-dimensional Penrose tilings Introduction to the Mathematics of Quasicrystals ed M V Jaric (New York: Academic) ch 4

[17] Katz A 1992 A short introduction to quasicrystallography From Number Theory to Physics ed M Wakdschmidt, P Moussa, J-M Luck and C Itzykson (Berlin: Springer) ch 11

[18] Joseph D, Baake M, Kramer P and Trebin H-R 1994 Diffusion in 2D quasicrystals Europhys. Lett, 27 45 I-56 